\title{
36. Die nordwestsemitischen Schriftkulturen
}

1. Allgemeines

2. Rahmenbedingungen

3. Textkorpus

4. Schule

5. Funktionen der Schrift

6. Schrift und Bild

7. Literatur

\section{Allgemeines}

Die Alphabetschrift, genauer: die westsemitische Konsonantenschrift, hat sich bereits in der 2. Hälfte des 2. Jahrtausends v. Chr. unter ägyptischem Einfluß einerseits, in Konkurrenz mit der Keilschriftkultur andererseits entwickelt und allmählich durchgesetzt. Da ihre Prinzipien der in Ugarit im 13. Jahrhundert v. Chr. verwendeten Keilalphabetschrift zugrundeliegen, muß sie damals bereits weiter verbreitet gewesen sein, als wir es bisher aufgrund der noch recht spärlichen Funde nachweisen können $(\rightarrow$ Art. 20). Aber erst nach den tiefgreifenden politischen Veränderungen am Übergang von der Spätbronze- zur Frühen Eisenzeit (12./11. Jahrhundert v. Chr.) 
findet die Linearschrift, wahrscheinlich von den phönizischen Städten ausgehend, weite Verbreitung und ausschließliche Verwendung. Sie wird im 10. Jahrhundert in Palästina (Gezer-Kalender), im 9. Jahrhundert im östlich angrenzenden Moab (Meša-Inschrift) und in den nördlich angrenzenden aramäischen Staaten übernommen und bald auch vermutlich im kleinasiatischen Raum - an. die Griechen weitergegeben. In dieser Zeit bleiben die allgemeinen Prinzipien dieser Schrift und ihrer Graphie unverändert:

- Schriftrichtung linksläufig im Gegensatz zur der Keilschrifttradition verhafteten Rechtsläufigkeit des Ugaritischen

- reine Konsonantenschrift (erst im aramäischen Kulturkreis kommt es zur Wiedergabe von Vokalen in best. Positionen, den matres lectionis)

- feste Buchstabenfolge in Abecedarien (zu Schulzwecken)

- Beibehaltung dieser Zeichenfolge (und der Zeichennamen) bei den Griechen.

Schon früher hatte sich die südsemitische Schrift als eigener Stamm mit gesonderter Entwicklung und einer anderen Konsonantenfolge abgespalten.

\section{Rahmenbedingungen}

\subsection{Schriftträger}

Die Entwicklung der Schriftkultur wird mit der Verbreitung der Schrift einhergegangen sein, doch wissen wir darüber - bedingt durch die Vergänglichkeit der Schriftträger nur wenig bzw. unser Bild der frühen Schriftkultur ist verzerrt. Zwar müssen wir davon ausgehen, daß vielerlei Materialien als Schriftträger gedient haben, doch hat das im Gegensatz zu Ägypten humide Klima im Mittelmeerraum und in Nordmesopotamien alle organischen Substanzen vernichtet. Folglich sind dort lediglich Texte erhalten geblieben, die auf Metall, Stein, Ton (Keramik), Verputz aufgemalt oder eingemeißelt wurden. Papyrus, Pergament, Holz, als Schreibmaterial sicher weithin in Gebrauch, sind verrottet oder verbrannt. Das gilt auch von den mit einer Wachspaste gefüllten Holz- oder Elfenbeintafeln, die mit Scharnieren zu Polyptycha zusammengefügt werden konnten (Funde in Nimrud und in Schiffswracks, z. B. Uluburun, Payton 1991). Die dafür übliche griechische Bezeichnung deltos geht sogar auf semitisch daltu (urspr. „Türflügel") zurück
(Galling 1971). Da diese Schriftträger, die zur Aufzeichnung ephemerer ebenso wie längerer und wertbeständigerer historischer, poetischer, religiöser Texte gut geeignet sind, entfallen, ist das Korpus der Texte stark beschränkt. Als Ausnahme müssen die Texte aus Ugarit gelten, die in einem eigenen Keilalphabet aufgezeichnet sind und deren Beschreibstoff, die Tontafel, sich verhältnismäßig gut erhalten hat.

\subsection{Schreibtechnik}

Die Schreibtechnik der uns erhaltenen Texte ist ziemlich einheitlich (Lemaire 1985). Ostraka, d. h. Gefäßscherben, wurden meist mit dem Pinsel bzw. der Binse und schwarzer Tinte beschrieben, zunächst auf der leicht gewölbten Vorderseite, erst wenn der Platz nicht ausreichte, auf der Rückseite. Dabei wurde das Ostrakon um seine Längsachse gedreht. Inschriften auf den Verputz von Wänden wurden ebenfalls mit (roter und schwarzer) Tinte geschrieben. Kurze Texte (Eigentums- bzw. Inhaltsvermerke) auf Gefäßen wurden auch vor oder nach dem Brand mit einem Stichel eingeritzt, selbst Stempel (bes. auf Krughenkeln) für wiederkehrende Vorgänge sind belegt. In die Tontafel, die als Schriftträger (abgesehen vom Ugaritischen) für die Aufzeichnung einer Linearschrift schlecht geeignet ist, wurde der Text eingeritzt, als Beischrift gelegentlich auch mit dem Pinsel aufgetragen. In Metallgegenstände (selten auch Elfenbein) wurde die Inschrift nach der Herstellung graviert, in besonderen Fällen (aramäische Inschriften auf Silberschalen) auch gepunzt. Steininschriften wurden normalerweise eingetieft, offenbar ohne vorherige Zeichnung auf dem Monument, nur in seltenen Fällen wurden die Buchstaben erhaben herausgearbeitet. Auf Papyrus und Pergament (z. B. Schriftrollen aus den Höhlen von Qumran am Toten Meer; $\rightarrow$ Abb. 36.1 auf Tafel V) wurde entweder mit der ägyptischen Binse oder mit der Rohrfeder geschrieben.

\subsection{Schriftgestaltung}

Die Schriftgestaltung kennt keine Unterschiede zwischen den einzelnen Textarten. Eine eigene Monumentalschrift wird nicht entwickelt, auch der Unterschied zwischen Groß- und Kleinbuchstaben existiert nicht. Natürlich sind Steininschriften formal steifer als die zur Kursive neigenden Tintenaufschriften der Ostraka und Papyri. Trotzdem ähneln die Buchstaben einander sehr, so daß paläographische Altersbestimmungen möglich 
sind. Regionale Unterschiede der Buchstabenformen bilden sich bereits im 9. Jahrhundert v. Chr. heraus und lassen bald Zuschreibungen zu bestimmten Kulturkreisen zu (Naveh 1982): Aramäisch mit der Tendenz der Öffnung der oberen Rundung einiger Zeichen; Hebräisch mit starken Ober- und Unterlängen, einer Tendenz zur Schräglage der Zeichen bei gleichzeitiger Abplattung; Punisch mit der Übernahme kursiver Unterlängen mit Verdickung in die Steininschriften, Herausbildung einer bald sehr vereinfachten „neupunischen“ Schriftvariante. Eine Besonderheit zeigt das Hebräische insofern, als es in nachexilischer Zeit, belegbar ab ca. 250 v. Chr., die eigene Schrifttradition verläßt und eine auf aramäischen Formen aufbauende "Quadratschrift" verwendet, die mit geringen Modifikationen bis heute in Gebrauch ist $(\rightarrow$ Art. 20, Zf. 4.1.2.). Sie kennt, wie auch später die arabische Schrift, bei einigen Buchstaben $(\mathrm{K}, \mathrm{M}, \mathrm{N}, \mathrm{P}, \mathrm{S})$ leicht abgewandelte Finalbuchstaben. Daß es eine bewußte „Neuerung“ war, läßt sich daran ablesen, daß der althebräische Gottesname YHWH zeitweilig noch ehrfürchtig mit den althebräischen Zeichen im sonst quadratschriftlichen Text erscheint. Schließlich verdient Erwähnung, daß kultische Texte in aramäischer Sprache auch in demotischer Schrift $(\rightarrow$ Art. 20, Zf. 1.1.3.) niedergeschrieben sein können (Vleeming \& Wesselius 1982; 1983/84; Nims \& Steiner 1983).

\section{Textkorpus}

Infolge der oben geschilderten Umstände ist das Textkorpus der Alphabetschriftkulturen bis in hellenistisch-römische Zeit hinein recht schmal. Es besteht im wesentlichen aus Steininschriften und Ostraka, in geringem Umfang auch in (in Ägypten gefundenen) Papyrusund Lederdokumenten, die sich zu Archiven zusammenfassen lassen. Die sehr zahlreichen punischen Weihinschriften aus Nordafrika besonders Karthago - und von den Inseln Malta, Sizilien und Sardinien sind wegen ihrer zweckgebundenen Stereotypie nur von beschränktem Wert. Ein Sonderfall ist das hebräische (und aramäische) Alte Testament (AT), das umfangreichste Dokument der frühen Alphabetschriftkultur, das aber in der uns vorliegenden Form recht jung und das Produkt tiefgreifender redaktioneller Veränderungen ist (s. 3.2.). Da es jedoch Textgattun- gen erhalten hat, die in allen übrigen Kulturen fehlen, muß es unbedingt berücksichtigt werden.

\subsection{Sprachen}

Im folgenden werden die Textgattungen ohne Rücksicht auf ihre sprachliche Zugehörigkeit behandelt, obgleich selbstverständlich sprachliche Identität auch kulturelle Identität begründet. Trotzdem ist es bei der verhältnismäßig schmalen Textbasis erlaubt, diese Differenzen gegenüber den Gemeinsamkeiten zurücktreten zu lassen. Sprachlich (und in gewissem Umfang auch graphisch) gehören die Dokumente drei Kulturkreisen an:

3.1.1. dem Kanaanäischen, wozu das Hebräische (in Palästina), das Moabitische, Ammonitische und Edomitische (im heutigen Jordanien) und das Phönizisch-Punische (Syrische Küste und Nordafrika) gehören;

3.1.2. dem Aramäischen, dessen altaramäische Sprachstufe besonders in Nordsyrien belegt ist, das sich als Verwaltungssprache des Achämenidenreiches (sog. Reichsaramäisch) über den ganzen Vorderen Orient vom 1. Katarakt in Ägypten bis zu den Dardanellen, nach Armenien und Iran verbreitete. Auch Teile des AT (Dan. 2,4-7,28; Ezra 4,8-6,18; 7,12-26; Jer. 10,11) und viele Texte vom Toten Meer (Beyer 1984) gehören dazu. Aus hellenistisch-römischer Zeit sind z. B. das Palmyrenische, Jüdisch-Aramäische und Nabatäische zu nennen. Das Syrische und weitere jüngere Sprachstufen bleiben hier außer Betracht;

3.1.3. dem Arabischen mit dem Altsüdarabischen (Sabäisch, Minäisch, Qatabanisch, Hadramautisch, Ausanisch; $\rightarrow$ Art. 21) und dem vorklassischen Nordarabischen, das sich z. T. im Nabatäischen, sonst im Thamudischen, Lichjanischen und Safaitischen fassen läßt. Hierfür ist allerdings der Bestand an Denkmälern extrem schmal. Da diese Denkmäler zwar dem gleichen Schrifttyp angehören, sprachlich aber vom Nordwestsemitischen zu trennen sind, werden sie nur am Rande berücksichtigt.

\subsection{Kanonbildung}

Während die meisten alphabetschriftlichen Texte keine Redaktionsarbeit erkennen lassen, hat das AT eine lange und nur noch teilweise rekonstruierbare Textgeschichte. Diese war erst mit der Arbeit der Masoreten 
ca. 750-1000 n. Chr. beendet, bei der der für den jüdischen Gottesdienst verbindliche hebräische Text des AT in seinem Konsonantentext mit Vokalzeichen, diakritischen Punkten, Lesezeichen, Hinweisen zur Rezitation usw. definitiv fixiert wurde, wobei noch unterschiedliche Schulen (Masoreten des Ostens bzw. des Westens, Kahle 1913; 1927-1930) nachweisbar sind. Diese sog. Masora legte aber bereits einen Text zugrunde, der als Ergebnis eines langen Entstehungsprozesses ca. um 200 v. Chr. festgelegt war. Das zeigt die griechische Übersetzung der sog. Septuaginta, die seit ca. 280 v. Chr. angefertigt wurde und die im wesentlichen, wenn auch mit manchen Umstellungen und Zusätzen, bereits den uns bekannten Text enthielt. Das gilt auch von den in Qumran gefundenen hebräischen Manuskripten des 1. Jahrhunderts v. Chr. Vorangegangen ist aber eine umfangreiche Redaktionsarbeit, in die wir nicht in allen Einzelheiten Einblick haben (Smend 1978). So wird angenommen, daß bei der Ausformung des Pentateuch am Anfang Einzelerzählungen standen, die teils als Sagen, teils als ätiologische Deutungen von Namen und Begriffen, teils auch als Bruchstücke von Stammes- oder Familiengeschichten überliefert und erst nach Überführung in die Schriftform zu größeren Kompositionen unter übergreifenden Gesichtspunkten (Erwählungstradition; Exodus; Landnahme usw.) zusammengefaßt wurden. Bei der Zusammenstellung ergaben sich Überschneidungen und Brüche, die es uns ermöglichen, einzelne Erzählkorpora (im Pentateuch z. B. Jahwist, Elohist und Priesterschrift) $\mathrm{zu}$ isolieren, deren jeweilige Zielsetzung sich noch wiedergewinnen läßt. Im Verlauf der Redaktionsarbeit kam es natürlich zu Diskrepanzen in den Aussagen, die die Redaktoren wieder durch z. T. erklärende $\mathrm{Zu}$ sätze auszugleichen suchten. Ferner scheinen manche Texte im Verlauf häufiger Abschreibarbeit verstümmelt und dann interpretierend verbessert oder von einzelnen Schreibern durch Glossen kommentiert worden zu sein, die danach in den Text einflossen und ihrerseits Erläuterungen erfuhren. Schließlich wurde der so entstandene Text im Rahmen einer Kultreform, die König Josia von Juda im Jahre 621 v. Chr. mit zentralistischer Tendenz (einziger Tempel ist jetzt der in Jerusalem) vornahm, in dem großen ,deuteronomistischen Geschichtswerk" nochmals von priesterlicher Seite überarbeitet. All das ist nur vorstellbar auf der Basis eines schriftlich fixierten, wohl in einzelnen Schriftrollen nie- dergelegten Textes, der wegen seines sakralen Charakters nur partielle Veränderungen zuließ. Ähnliches gilt auch von prophetischen Texten. So ist das dem Propheten Jesaja, der im 8. Jahrhundert v. Chr. wirkte, zugeschriebene Buch aus mindestens 4 Quellen zusammengestellt: 1. Der eigentliche Jesaja mit seinen prophetischen Sprüchen (Kap. 1-35), 2. den Erzählungen (Kap. 36-39), die einen Bericht aufnehmen, der bereits in 2. Reg. 18,13 bis $20,19 \mathrm{zu}$ finden ist, 3. Deuterojesaja (Kap. 40-55), der erst in der Achämenidenzeit geschrieben haben kann, und 4. Tritojesaja (Kap. 56-66), dessen Wirken um die Zeit des Exils (d. h. nach 587) angesetzt wird.

Viele Bücher des AT sind unter textkritischen Gesichtspunkten also zunächst ganz uneinheitlich gewesen. Sie wurden aber in einem theologischen und literarischen Formungsprozeß allmählich zu den Einheiten verschmolzen, als die sie bereits in hellenistischer Zeit den Kanon bildeten. Abgesehen von Apokryphen gibt es aber in den kanonischen Texten selbst genügend Hinweise auf ursprünglich vorhandene Quellen, die völlig ausgemerzt oder nur in stark verkürzter Form überliefert wurden. All dies ist nur denkbar auf dem Hintergrund einer breiten schriftlichen Überlieferung, die von priesterlichen Schreibern weitergeführt, exzerpiert und redigiert wurde. Schriftlichkeit der Vorlagen erweist sich auch an zahlreichen ,Verbesserungen“ und daraus folgenden Uminterpretationen von offenbar korrupten Passagen einer Vorlage, die von den jeweiligen Abschreibern vorgenommen wurden. Sie lassen sich $u$. a. an Textvarianten ablesen, die in der griechischen Übersetzung des AT (sog. ,Septuaginta“) erscheinen, folglich einen Text repräsentieren, der um die Mitte des 3. Jahrhunderts v. Chr. zumindest zum Teil niedergeschrieben wurde (Würthwein 1988).

\section{Schule}

\subsection{Schreiber}

Die allenthalben feststellbare Uniformierung der Schriftform und der Graphie setzt eine Schulung voraus, die durch Schreiber, die als eigener Berufsstand existierten (Bonnet 1991), vermittelt wurde. Die Funde von Abecedarien (bereits in Ugarit) und typischen „Schultexten" in Linearschrift belegen eine solche Praxis schon für das 13. Jahrhundert v. Chr. Verschiedene Anspielungen im AT lassen ein System der Erziehung, zu dem auch Lesen 
und Schreiben gehörte, erschließen (Lemaire 1981). Danach gab es zumindest bestimmte Berufszweige (u. a. Priester und Propheten), die des Lesens und Schreibens kundig waren. Obgleich die Alphabetschrift gegenüber der Keilschrift oder den Hieroglyphen sicher leichter zu erlernen war, ist doch keine Gewißheit darüber zu gewinnen, in welchem Umfang sie allgemein gelesen und geschrieben werden konnte. Die beachtliche Zahl von „öffentlichen" Inschriften, d. h. königlichen Verlautbarungen (Meša-Stele KAI Nr. 181; Karatepe-Inschrift KAI Nr. 26 usw.) oder juristischen Dokumenten, z. B. Verträgen (SfireInschrift KAI Nr. 222-224) oder Steuertarifen (Palmyra, CIS II 3913) läßt allerdings darauf schließen, daß die Zahl der möglichen Leser nicht klein war. Zumindest unter den höheren Beamten war, nach den auf Ostraka erhaltenen Briefen zu urteilen, die Lesefähigkeit verbreitet und wurde auch in Schulen gelehrt.

\subsection{Graphische und sprachliche Formung}

Es ist deshalb auch nicht verwunderlich, daß gewisse Schreibgewohnheiten allgemein verbreitet sind. Die Texte werden fast immer fortlaufend geschrieben. Worttrenner als Punkte (und selten auch kurze Striche zur Abteilung von Phrasen) sind nur sehr früh und dann wieder spät (unregelmäßig) in Gebrauch, Spatien zur Trennung von Wörtern sind zunächst unbekannt, Wortende und Zeilenende müssen nicht übereinstimmen. Gelegentlich werden Absätze (oder Zwischenstriche) zur Gliederung längerer Texte verwendet. Vor allem Verwaltungstexte haben einen streng eingehaltenen Aufbau, sie sind, je nach Inhalt, z. B. in Kolumnen gegliedert, setzen eine genormte Verwaltungspraxis voraus. Dasselbe gilt von Briefen, die meist einem festen Formular folgen (Pardee 1982). Auch die Bittschrift eines Erntearbeiters (KAI Nr. 200) läßt erkennen, daß bestimmte juristische Vorgänge formalisiert waren und in Schriftform erledigt werden mußten. An einen Leser allein wenden sich Texte des AT (Ps. $9+10$, $25,34,37,111,112,119,145$. Klagelieder 1-4), die nach dem System des Akrostich aufgebaut sind, die hebräische Alphabetfolge als Zeilenbeginn erkennen lassen.

\footnotetext{
4.3. Archive und Bibliotheken

Die Existenz von Archiven für Texte der Verwaltung oder der Jurisdiktion ist schon früh nachweisbar: In Ugarit sind zusammengehörige Vorgänge an einer Stelle gelagert gewe-
}

sen; der König von Byblos läßt im Reisebericht des Ägypters Wen-Amun (um 1100 v. Chr.). ,die Tagesrollen seiner Väter“ holen, um zurückliegende Zahlungen der Ägypter festzustellen; ganze zusammengehörige Komplexe von Ostraka sind z. B. in Samaria, Arad und Lachisch gefunden worden, wobei die Fundsituation nahelegt, daß die entsprechenden Vorgänge zwar gemeinsam ,abgelegt“ waren, dann aber weggeworfen wurden. Die jüdische Militärkolonie in Elephantine (Oberägypten) hatte sowohl private als auch offizielle Archive; in letzteren wurden auch die Abschriften und Entwürfe von Schreiben aufbewahrt, die nach Jerusalem gegangen waren (Porten 1968). In Karthago wurde unlängst in der Nachbarschaft eines Tempels ein riesiges Lager von Tonbullen mit Siegelabdrükken entdeckt, die zu einem verbrannten Archiv von Papyrusurkunden gehört haben müssen, das über mehrere Jahrhunderte lief. Regelrechte Bibliotheken, d. h. systematisch gesammelte und thematisch geordnete Bestände an Büchern bzw. Schriftrollen, lassen sich für vorhellenistische Zeit nicht nachweisen. Wenn z. B. in 2. Reg. 22,8 die Rede davon ist, daß „das Gesetzbuch im Tempel des Herrn gefunden" wurde, so deutet dieser offenbar „zufällige“ Fund darauf hin, daß es auch dort keine Bibliothek gab, wohl aber Archive, die auch für den Kultus angelegt wurden (evtl. im Sinne einer Geniza). Für achämenidische und spätere Zeit sind wieder im AT Hinweise auf Büchersammlungen erhalten (Kellermann 1982). Die umfangreiche punische Literatur (Huss 1985, 504 ff) war in Tempelbibliotheken gesammelt und wurde nach der Eroberung Karthagos von den Römern den numidischen Fürsten übergeben (Plinius, Hist. nat. 18,22).

\section{Funktionen der Schrift}

\subsection{Wirtschaft und Verwaltung}

Wenn das AT schon für die Zeit Davids und Salomos eine differenzierte Beamtenschaft an der Staatsspitze nennt (2. Sam. 8,16-18; 20,23 - 26; 1. Chron. 27,25-34; 1. Reg. 4,719), so dürfen wir annehmen, daß dem eine wohl unter ägyptischem Einfluß in Kanaan bereits früher entstandene Organisation entsprach, die auch verschriftete Verwaltungsakte voraussetzt. Ein Großteil der uns als Ostraka erhaltenen Dokumente frühen Schriftgebrauchs betrifft denn auch Vorgänge wie den Eingang und Ausgang von Waren als Lieferungen (teilweise von Krongütern) an den $\mathrm{Pa}$ - 
last oder Vergabe von Rationen. Sie haben eine knappe, dem Sachverhalt angemessene Diktion, nennen selten mehr als das Quantum, das Gut (gelegentlich noch mit Qualitätsmerkmalen), den Lieferanten oder Empfänger, manchmal noch den Herkunfts- oder Bestimmungsort und ein Datum. Daneben können diese Texte auch als reine Personenlisten gestaltet sein. Zunächst aus Ugarit, dann wieder aus dem Elephantine-Archiv des 6. Jahrhunderts v. Chr. (Porten 1968) sind Urkunden über Grundstückstransaktionen, Erbschaften, Mitgift, Testamente usw. bekannt, die bereits eine sehr spezifische Terminologie verwenden. Das läßt darauf schließen, daß sie in einer längeren Tradition stehen, die uns nicht überliefert ist. Dabei ist daran zu erinnern, daß in den Keilschriftkulturen Recht prinzipiell der Schriftform bedurfte, so daß Ähnliches auch in Kanaan gegolten haben wird. - In den gleichen Kontext gehören die meisten Briefe, die in der Regel keine persönlichen Anliegen zum Inhalt haben, sondern Wirtschaft, Verwaltung oder auch (z. B. KAI Nr. 266) politisch-historische Fragen betreffen.

\subsection{Geschichte und Tradition}

„Was sonst noch von $\mathrm{PN}$ zu sagen ist, alles was er getan hat, siehe, das steht geschrieben in der Chronik der Könige von Israel (bzw. von Juda)". Dieser in den Königsbüchern häufige Satz verweist auf eine Hofchronik, die offenbar den Büchern der Könige bzw. Chronik des AT zugrunde gelegen hat, uns aber nicht erhalten ist. Vergleichbares wird von dem Phönizier Sanchuniaton (bei Euseb von Caesarea, Praep. Evang. I 9,20) gesagt, nämlich daß er ,die ganze alte Geschichte aus den Überlieferungen der einzelnen Städte und den in den Heiligtümern vorhandenen Aufzeichnungen zusammengestellt" habe. Die historische Tradition war also zumindest in einer Art Annalistik niedergeschrieben. Analogien liefern babylonische und assyrische historische Berichte, die als Eponymen- oder Königslisten in Kurzform, als jährliche Chroniken wesentlich ausführlicher die für wichtig gehaltenen Ereignisse verzeichnen (vgl. Grayson 1975). Vermutlich nehmen die historischen Texte des AT jedoch eine Sonderstellung ein. Sie erheben sich weit über die reine Annalistik, sind mit ihren vielerlei Kunstmitteln und Reflektionsebenen, mit ihrer bewußt theologischen Begründung bzw. Kommentierung von Handlungen bereits Geschichtsschreibung in unserem Sinne (Cancik 1976).
Erhalten sind sonst nur wenige Bau- oder Grabinschriften von Herrschern (z. B. Bauinschr.: KAI Nr. 4 [Jehimilk v. Byblos], Nr. 7 [Šipițba al I.], Nr. 10 [Jehaumilk], Nr. 15.16 u. a. [Bodaštart], Nr. 24 [Kilamuwa], Nr. 26 [Azatiwada]; Grabinschr.: KAI Nr. 1 [Ahīrōm v. Byblos], Nr. 9 [Sohn des Šipița al III.], Nr. 11 [Batno am], Nr. 13 [Tabnit], Nr. 14 [Ešmunazar] usw.). Diese sind durchaus literarisch geformt: Auf Nennung des Objekts folgt der Name des Stifters, Angabe des Zwecks bzw. Hinweis auf eine Weihung, Segensformel für den Stifter und Fluchformel gegen einen möglichen Veränderer des Bauwerks. Die Kilamuwa-Inschrift $(\rightarrow$ Abb. 20.4 auf Tafel V) hat darüber hinaus eine durchaus poetische Struktur (O'Connor 1977).

\subsection{Mythos, Ritus und Kultus}

Hier ist der Textbestand, gemessen etwa am benachbarten Ägypten oder Mesopotamien, wieder sehr beschränkt. Während Mythen z. B. aus Ugarit in beachtlicher Zahl bekannt sind (Caquot, Sznycer \& Herdner 1974), ist das vergleichbare Material der Phönizier unter dem Namen des Priesters Sanchuniathon nur in vielfach gebrochener Tradition ganz bruchstückhaft nach Philo von Byblos bei Euseb von Caesarea erhalten (Ebach 1979). Während von althebräischer Mythologie sich Reste im AT erhalten haben (Schöpfungsgeschichte(n), Sintflut, Götterkampf), sind keinerlei vergleichbare Texte aus dem aramäischen und arabischen Kulturkreis erhalten geblieben. Religiöse Riten hat es, folgt man Anspielungen in antiken Autoren, viele gegeben, z. B. um Adonis, Melqart usw., doch sind auch hier originale Aufzeichnungen verloren. Für den großen Bereich der Magie sind lediglich zwei Beschwörungen (KAI Nr. 27 und Teixidor 1983) und einige Fluchtäfelchen (z. B KAI Nr. 89) bekannt. Sie unterstreichen den magischen Charakter des geschriebenen Wortes, da sie, amulettartig ins Grab und damit in die Unterwelt gebracht, von dort aus noch lebenden namentlich genannten Personen Unglück bringen sollten. - Zum Kult gehören die zahllosen stark formalisierten Weihinschriften auf Steinstelen, vor allem aus Nordafrika (bes. Karthago), die mit Hinweis auf ein Opfer (oft eines Kindes oder seines Ersatzes) die göttliche Hilfe erflehen (Beispiele KAI Nr. 61. 63. 64. 84-88. 102-112). Grabstelen enthalten z. T. Formeln, die sich auf den Totenkult beziehen (Schmuck der Toten, Gesichtsmasken, Libationen). Außerordentlich reich ist dagegen die kultische Über- 
lieferung des AT, auch wenn durch die deuteronomistische Redaktion nachträglich eine Bereinigung und Ausrichtung lediglich auf den Kult von Jerusalem vorgenommen wurde. Die kultischen Vorschriften besonders des Buches Leviticus regeln nicht nur den Tempeldienst, sondern auch die Bestallung von Priestern, die großen Festlichkeiten und selbst das individuelle Verhalten bei bestimmten täglichen Verrichtungen (Speisegebote usw.). Viele der im AT überlieferten Psalmen und Lieder haben ihren festen Sitz in diesen Festtagsliturgien besessen. Vergleichbares ist von den Nachbarkulturen anzunehmen, aber verloren. - Prophetische Texte, wiederum im AT reichlich erhalten, hat es auch in den Nachbarkulturen gegeben. Ein leider schlecht erhaltener Text ist das Bileams-Orakel aus Dēr 'Allā (Weippert 1991).

\subsection{Erzählung, Lied, Spruch und Texte zur Weisheit}

Erzählliteratur hat wieder nur das AT erhalten, z. B. in den Patriarchen-Erzählungen, den Geschichten über Joseph, über Mose und - in etwas abgewandelter Form - im Buch Esther. Sie sind auch in den eher historisch orientierten Büchern zu finden, so etwa die Geschichte von der Thronfolge Davids (2. Sam. $13-20$ und 1. Reg. 1-2). Sie sind, wie etwa die Josephsgeschichten, in der überlieferten Form kompliziert gebaute literarische Kompositionen (Schweizer 1991), gehen aber im Kern auf mündliche Berichte zurück, die erst im Prozeß $ß$ der Verschriftlichung ihre anspruchsvolle Form erhielten. Darüber hinaus hat das AT in unterschiedlichen Textzusammenhängen Lieder erhalten, die offenbar in den Nachbarkulturen nicht verschriftet worden sind, von denen sich jedenfalls sonst in den Zeugnissen der frühen Alphabetschrift keine Reste gefunden haben. Es sind dies Arbeits-, Ernte- und Trinklieder (Num. 21,17f; Jes. 22,16; Sap. Sal. 2,1-20); Hochzeits- und Liebeslieder (Sog. Hohelied); Wächterlied (als Anspielung in Jes. 52,8 f); Spottlieder (Num. 21,27-30; Jes. 37,22-29) und Leichenlieder (2. Sam. 1,19-27; 3,33 f; Jes. 14,4-21). Auch Klagelieder und Danklieder (Ps. 66,2-12 bzw. 13-20 u. ö.) sind Gattungen, die nur im AT in die Schriftlichkeit überführt worden sind. Sie setzen einen „Literaturbetrieb“ voraus, der aus inhaltlichen und kompositorischen Gründen literarische Gattungen in die Schriftform überführte, die ursprünglich nicht für eine schriftliche Tradierung bestimmt waren. Es ist deshalb auch nicht verwunder- lich, daß die anderen Schriftkulturen der Region nichts davon bewahrt haben. Anders verhält es sich mit Weisheitstexten, die in Ägypten und Mesopotamien selbstverständlicher Bestandteil der schriftlichen Tradition waren ( $\rightarrow$ Art. 20, 2.3.2.) und natürlich reichlich im AT (Hiob-Dichtung; Qohelet; Sapientia Salomonis) vertreten sind, aber auch in Form des sog. Achiqar-Romans mit Rahmenhandlung und Spruchsammlung eine reichsaramäische Überlieferung kennt (Lindenberger 1983).

\subsection{Repräsentation}

Fragen wir nach den Motiven von Schriftlichkeit in den frühen Jahrhunderten der Verwendung der Alphabetschrift, so ist das Bedürfnis zur Selbstdarstellung, zur Verewigung der eigenen Taten sicher ein bevorzugter Anlaß gewesen. Zahllose Felsinschriften einfachster Form - besonders im arabischen Sprachraum - enthalten selten mehr als den Namen und Vatersnamen des Schreibenden, gelegentlich mit der Anrufung einer Gottheit verbunden, oft aber lediglich mit dem Hinweis auf kurzzeitiges Verweilen an diesem Ort. Diese Bekundung hat dann spätere Besucher veranlaßt, sich in die Nachfolge zu einer solchen Präsenz einzureihen. Umfangreicher als diese Privatinschriften sind die vergleichbaren von Königen, die oft noch best. Bauten, Weihungen und andere Taten zu ihrem Ruhme und zur Erinnerung für die Nachfahren anführen. Auch Grabinschriften verfolgen ähnliche Ziele. Der magische Charakter des Geschriebenen wird dabei auch eine Rolle gespielt haben, doch wird dies nur in dem Verbot der Namenstilgung in der Fluchformel spürbar. Ein besonderer Typ der Schrift ist zur Repräsentation z. B. auch auf Siegeln (Sass \& Uehlinger 1993) nicht geschaffen worden.

\section{Schrift und Bild}

Die Alphabetschrift hat aufgrund des geringen dekorativen Charakters ihrer Zeichen im Gegensatz zu ägyptischen Hieroglyphen, chinesischen Wortzeichen usw. im allgemeinen keine spezifisch künstlerische Verwendung gefunden. Lediglich altsüdarabische Inschriften treten gelegentlich auch als Gebäudeschmuck hervor. Andererseits lassen sich kurze Alphabettexte leicht auf kleinen Schriftträgern (z. B. Münzen) anbringen, ohne die Darstellung zu stören oder indem sie deren Komposition abrunden. Als Beischriften z. B. auf Grabdenk- 
mälern (u. a. in Palmyra), auf den Sockeln von Bildnissen, neben Darstellungen auf Wandgemälden usw. machen sie mit wenigen Zeichen die Identifikation des Dargestellten möglich, so daß für den Schriftkundigen neben die ikonographische Charakterisierung noch die durch die Schriftsprache tritt.

\section{Literatur}

Barucq, A., Caquot, A., Durand, J. M., Lemaire \& Masson, E. 1986. Ecrits de 1'Orient Ancien et sources bibliques, Paris.

Bonnet, Corinne. 1990. La terminologie phénicopunique relative au métier de lapicide et à la gravure des textes. SEL 7, $111-120$.

Beyer, Klaus. 1984. Die aramäischen Texte vom Toten Meer. Göttingen.

Caquot, Andrè, Sznycer, Maurice \& Herdner, Andrée. 1974. Textes ougaritiques I. Mythus et Légends. Paris.

Cancik, Hubert. 1976. Grundzüge der hethitischen und alttestamentlichen Geschichtsschreibung. Abhdlg. Dt. Palästinavereins: Wiesbaden.

CIS. Corpus Inscriptionum Semiticarum. Paris.

Donner, Herbert \& Röllig, Wolfgang. 1966. Kanaanäische und aramäische Inschriften. (KAI) Bd. 1-3. 2. Aufl. Wiesbaden.

Ebach, Jürgen. 1979. Weltentstehung und Kulturentwicklung bei Philo von Byblos. Beitr. zur Wiss. vom Alten u. Neuen Testament 108. Stuttgart.

Galling, Kurt. 1971. Tafel, Buch und Blatt. In: H. Goedicke (ed.). Near Eastern Studies ... W. F. Albright, Baltimore/London.

Grayson, Kirk Albert. 1975. Assyrian and Babylonian Chronicles. Texts from Cuneiform Sources 5. Locust Valley, N. Y.

Huss, Werner. 1985. Geschichte der Karthager. Handb. d. Altertumswissensch., Abt. III Vol. 8. München.

Kahle, Paul. 1913. Masoreten des Ostens. Leipzig. -. 1927. 1930. Masoreten des Westens. I. II. Stuttgart.

KAI s. Donner \& Röllig 1966.

Kellermann, Mechthild. 1982. „Wenn ihr nun eines von diesen Büchern braucht, so laßt es euch holen." (2. Makk. 2,15). Zeitschrift des Deutschen Palästina-Vereins 98, 104-109.

Lemaire, André, 1981. Les écoles et la formation de la Bible dans l'Ancien Israel. Orbis Biblicus et Orientalis 39. Fribourg/Göttingen.
-. 1985. Vom Ostrakon zur Schriftrolle, in: W. Röllig (ed.), Zeitschr. der Dt. Morgenländ. Gesellschaft, Suppl. 6, 110-123.

Lindenberger, J. M. 1983. The Aramaic Proverbs of Ahiqar. Baltimore - London.

Naveh, Joseph. 1982. Early History of the Alphabet. Jerusalem/Leiden.

Nims, C. F. \& Steiner, R. C. 1983. A Paganized Version of Psalm 20,2-6 from the Aramaic Text in Demotic Script. Journal of the American Oriental Society 103, 261-274.

O'Connor, M. 1977. The Rhetoric of the Kilamuwa Inscription. Bull. American Schools of Oriental Research 226, 15-29.

Pardee, Denis. 1982. Handbook of Ancient Hebrew Letters. Sources for Biblical Study 15. Chico/California.

Payton, Robert. 1991. The Ulu Burun Writing Board Set. Anatolian Studies 41, 99-106.

Porten, Jezabel. 1968. Archives from Elephantine. Berkely/Los Angeles.

Sass, Benjamin \& Uehlinger, Christoph (ed.). 1993. Studies in the Iconography of Northwest Semitic Inscribed Seals. Orbis Biblicus et Orientalis 125. Fribourg/Göttingen.

Schweizer, Harald. 1991. Die Josefsgeschichte. Konstituierung des Textes. Bd. 1. 2. Tübingen.

Smend, Rudolf. 1978. Die Entstehung des Alten Testaments. Stuttgart.

Teixidor, Javier. 1983. Les tablettes d'Arslan Tash au Musée d'Alep. Aula Orientalis 1, 105-108.

Tropper, Josef. 1993. Die Inschriften von Zincirli. Münster.

Vleeming, S. P. \& Wesselius, J. W. 1982. An Aramaic Hymn from the Fourth Century B. C. Bibliotheca Orientalis 39, 501-509.

-. 1983/84. Betel the Saviour. Jaarbericht ex Oriente Lux 28, 110-140.

Weippert, Manfred. 1982. Die „Bileam“-Inschrift von Tell Dēr 'Allā. Zeitschrift des Deutschen Palästina-Vereins 98, 77-103.

-. 1991. The Balaam Text from Deir Alla and the Study of the Old Testament, in: J. Hoftijzer \& G. van der Kooij (ed.). The Balaam Text from Deir Alla Re-evaluated. Leiden.

Würthwein, Ernst. 1988. Der Text des Alten Testaments. 5. Aufl. Stuttgart.

Wolfgang Röllig, Tübingen (Deutschland) 